\title{
Escuta de Crianças com Fissura Labiopalatina na Escola
}

\section{Children's Listening with Cleft Lip and Palate in the School}

\author{
Rosana Ribeiro Manoel*, Mariza Ribeiro Feniman**, Maria José Monteiro Benjamin Buffa***, \\ Luciana Paula Maximino****, José Roberto Pereira Lauris *****, José Alberto de Souza Freitas******. \\ * Doutora em Ciências da Reabilitação - Área de Distúrbios da Comunicação do Hospital de Reabilitação de Anomalias Craniofaciais da Universidade de São Paulo - HRAC- \\ USP. Chefe de Seção do HRAC-USP, Bauru-SP. \\ ** Pós-Doutorado em Audiology - Univeristy of Cincinnati-Ohio-USA. Chefe de Departamento de Fonoaudiologia-FOB-USP. \\ **** Mestre em Distúrbios da Comunicação Humana pelo HRAC-USP. Pedagoga - HRAC-USP. \\ ***** Doutora pelo Instituto de Biociências da Universidade Estadual Paulista - UNESP-Botucatu-SP. Professora Doutora do Departamento de Fonoaudiologia da \\ FOB-USP. \\ ****** Livre-Docência. Professor Associado do Departamento de Odontopediatria, Ortodontia e Saúde Coletiva da FOB-USP. \\ ******* Professor Titular do Departamento de Estomatologia da FOB-USP. Superintendente do HRAC-USP. \\ Instituição: Hospital de Reabilitação de Anomalias Craniofaciais - HRAC-USP. \\ Bauru / SP - Brasil \\ Endereço para correspondência: Mariza Ribeiro Feniman-Faculdade de Odontologia de Bauru da Universidade de São Paulo-FOB-USP - Departamento de Fonoaudiologia/ \\ FOB-USP - Alameda Octavio Pinheiro Brisolla 9-75 - Bauru / SP - Brasil - CEP: 17012-101 - Telefone: (+55 14) 3225-8533 - E-mail: feniman@usp.br \\ Artigo recebido em 31 de Março de 2010. Artigo aprovado em 23 de Abril de 2010.
}

\section{RESUMO}

Introdução:

Objetivo:

Método:

Resultados:

Conclusão:

Palaviras-chave:

\section{SUMMARY}

Introduction:

Method:

Results:

Conclusion:

Keywords:
Grande similaridade entre o comportamento de pacientes com fissura labiopalatina e aqueles com transtorno de processamento auditivo são relatadas por pais e professores.

Verificar a escuta de crianças com fissura labiopalatina em seis condições de escuta.

Professores de 224 escolares (7 a 11 anos) com fissura completaram um questionário, visando julgar a escuta do escolar no ruído, condição ideal, com múltiplos estímulos, no silêncio, quando solicitado recordar a informação ouvida e durante longo período de escuta, comparando-o ao de outro sem fissura de mesma idade e condição de escuta. Estudo Prospectivo.

A média do julgamento $(-0,08$, desvio padrão de 0,27$)$ dos alunos com fissura, realizado pelo professor, foi aproximadamente ao de "mesma dificuldade" (zero), quando comparado com o escolar sem fissura. Não foi encontrada significância estatística para qualquer uma das condições, nem para o valor total do questionário considerando os gêneros e as séries escolares.

As características de escuta dos escolares com fissura labiopalatina foram similares ao de outro sem esta malformação craniofacial de mesma idade e condição de escuta semelhante. No ruído, quando a memória e a atenção auditiva são requeridas foram as condições mais difíceis.

criança, fissura palatina, audição, percepção auditiva.

A great similarity between the patients with cleft lip and palate' behavior and those with auditory processing disorder are related by parents and professors. Objective: To verify the listening in children with cleft lip and palate in six conditions of listening.

Professors of 224 students ( 7 to 11 years old) with cleft completed a questionnaire aiming to judge the student listening in the noise, ideal condition, with multiple stimulus, in the silence, when it is solicited to remember the listened information and during a lengthy period of listening, comparing it to the other of the same age and listening condition, without cleft. A Prospective Study.

The mean of the trial $(-0,08$, standard deviation of 0,27$)$ of the students with cleft, performed by professor was about the "same difficulty" (zero), when compared with the student without cleft. It was not found statistical significance to anyone conditions, neither to the total value of the questionnaire, considering the gender nor the school year level.

The listening characteristics of the students with cleft lip and palate were similar to the other without this craniofacial deformity of the same age and similar listening condition. In the noise, the conditions more difficult occurred when the memory and the auditory attention were required.

child, cleft palate, hearing, auditory perception. 


\section{INTRODUÇÃO}

Grande similaridade entre o comportamento de pacientes com fissura labiopalatina e aqueles com transtorno de processamento auditivo foram relatadas por pesquisadores investigando as habilidades auditivas, por meio da aplicação de um questionário (1) e de testes comportamentais (2).

Transtorno de processamento auditivo refere às dificuldades no processamento perceptual de informação auditiva no sistema nervoso central. O problema pode ser exacerbado em ambientes acústicos desfavoráveis $(3,4)$. Desta forma, crianças com este transtorno são descritas pelos seus pais e professores como tendo dificuldade de escutar na presença de ruído de fundo, em seguir instruções orais, assim como, terem dificuldade de entender a fala distorcida, a fala na presença de dois falantes, como por exemplo, em situação de discussão em grupo (3). Este transtorno é apontado como um dos fatores das 50\% das crianças brasileiras, segundo dados do Ministério da Educação (MEC), que chegam ao final do ensino médio com sérios problemas de leitura e escrita (5). Assim, atualmente, a associação entre dificuldades escolares e alterações no desenvolvimento de habilidades auditivas tem sido enfatizada em estudos realizados com testes de processamento auditivo (6). Pesquisadores (7) relataram que crianças com dislexia apresentam alterações do processamento neurológico central que podem ser detectadas tanto em testes específicos de processamento auditivo, quanto em exames funcionais de imagem como SPECT (Single Photon Emission Computed Tomography).

Uma variedade de procedimentos informais comportamentais, elaborados para pais e/ou professores, tem sido desenvolvido para, sistematicamente, investigar comportamentos que podem ser úteis em determinar se uma criança deve ser encaminhada para uma avaliação de processamento auditivo $(3,8)$.

A literatura (9) tem demonstrado que muitas informações de pais/e ou professores ajudam a identificar alterações encontradas em suas crianças.

O presente estudo tem como objetivo verificar a escuta de crianças com fissura labiopalatina, por meio do julgamento do professor observando as características de seus alunos com este tipo de malformação craniofacial no silêncio, em situação de escuta ideal, na presença de múltiplos estímulos, no ruído, quando solicitada a recordar a informação ouvida e, em longo período de escuta.
Tabela I. Distribuição das crianças quanto à idade e ao gênero.

\begin{tabular}{lcccccc}
\hline Gênero & 7 & \multicolumn{7}{c}{ Idade (anos) } \\
& 7 & 8 & 9 & 10 & 1 I & Total \\
\hline Masculino & 17 & 29 & 46 & 38 & 11 & $14 \mid$ \\
Feminino & 18 & 17 & 27 & 16 & 5 & 83 \\
\hline Total & 35 & 46 & 73 & 54 & 16 & 224 \\
\hline
\end{tabular}

\section{MÉTODO}

A fim de se compor à casuística desse estudo de coorte contemporânea com corte transversal, selecionou-se pacientes com o tipo de fissura de maior ocorrência em um Hospital especializado nesta malformação craniofacial. Por isso, para o universo de 800 crianças com fissura envolvendo o lábio e palato à esquerda, operadas, de ambos os gêneros, que se encontravam na faixa etária entre 7 a 11 anos, regularmente matriculadas no Hospital, e, que cursavam classe regular, em nível de $1^{\underline{a}}$ a $4^{\underline{a}}$ série foi enviado, via correio, um questionário para ser entregue a seus professores, visando o objetivo proposto neste estudo. Um mesmo professor pode ter trabalhado com mais de uma criança em diferentes salas de aula.

Orientações quanto ao objetivo da pesquisa, ao preenchimento do questionário foram fornecidas, por escrito, aos professores. Retornaram respondidos 224 questionários, juntamente com o termo de consentimento livre e esclarecido assinado. Assim, participaram deste estudo prospectivo professores de 141 crianças do gênero masculino e 83 do feminino e idade média de 9 anos.

A Tabela 1 apresenta a distribuição das crianças quanto à idade e ao gênero.

O questionário aplicado e respondido foi o CHAPPSChildrens Auditory Processing Performance Scale (8), (Figura 1a e 1b) que foi desenvolvido para coletar e quantificar sistematicamente as características de escuta de crianças. É um questionário tipo escala das características de escuta de crianças. Tem como objetivo verificar o julgamento do professor das características de escuta de escolares em seis condições/funções de escuta: em ambiente silencioso, no ruído, quando é requerido lembrar a informação ouvida (memória auditiva/sequência) e, em longos períodos de escuta (atenção auditiva).

Este instrumento foi escolhido, por nestas condições de escuta estarem as queixas mais frequentes mencionadas na literatura (8-10), por pais e professores de alunos que apresentam transtorno do processamento auditivo. 


\section{CHAPPS-Children's Auditory Processing Performance Scale Smoski et al. 1992}

Nome da criança:

Data de nascimentos

Escolaridade (série):

Informante:
Data:

Idade atual:

\section{POR FAVOR, LEIAAS INSTRUÇÕES CUIDADOSAMENTE}

Responder todas as questões pela comparação desta criança às outras de idade e escolaridade similares. Não responder as questões baseado apenas na dificuldade da condição de escuta. Por exemplo, todas as crianças de 8 anos de idade, até certo ponto, podem não ouvir e entender quando estão em uma sala de aula ruidosa. Isto seria uma condição difícil de escutar para todas as crianças. Entretanto, algumas crianças podem ter mais dificuldades nessa situação de escuta do que outras. Você deve julgar se esta criança tem ou não MAIS dificuldade que as outras em cada condição de escuta citada. Favor marcar com um X em cada item seu julgamento, usando as seguintes respostas.

\section{MENOS DIFICULDADE \\ MESMA DIFICULDADE MAIS DIFICULDADE}

\section{Condição de escuta - RUÍDO}

Se esta criança estiver escutando em uma sala onde exite ruído de fundo, tais como TV, música, outras pessoas conversando, crianças brincando, etc., esta criança tem dificuldade de ouvier e entender (comparando a outras crianças da mesma idade e escolaridade)

1. Se esta criança estiver prestando atenção ela terá

2. Se for feita uma pergunta para esta criança ela terá

3. Se for dada uma instrução simples para esta criança ela terá

4. Se for dada instruções complexas e variadas esta criança terá

5. Se esta criança não estiver prestando atenção ela terá

6. Se esta criança estiver desenvolvendo outra atividade como lendo, colorindo, etc. ela terá

7. Se esta criança estiver em grupo com outras criança, ela terá

Grau de Dificuldade
Menos
$\begin{array}{ccc}(\text { Mesma Mais } \\ (\text { ) } & (\text { ) } & (\text { ) } \\ (\text { ) } & (\text { ) } & (\text { ) } \\ (\text { ) } & (\text { ) } & (\text { ) } \\ (\text { ) } & (\text { ) } & (\text { ) } \\ (\text { ) } & (\text { ) } & (\text { ) } \\ (\text { ) } & (\text { ) } & (\text { ) }\end{array}$

Condição de escuta - SILENCIOSA:

Se esta criança estiver escutando em uma sala silenciosa (outros podem estar presentes, mas estão quietos), esta criança tem dificuldade de ouvir e entender (comparado com outra criança).

Grau de Dificuldade

Menos Mesma Mais

8. Se esta criança estiver prestando atenção ela terá

9. Se for feita uma pergunta para esta criança ela terá

10. Se for dada uma instrução simples para esta criança ela terá

11. Se for dada instruções complexas e variadas esta criança terá

$($ ) ( ) ( )

$($ ( ) $\quad($ )

( ) ( ) ( )

12. Se esta criança não estiver prestando atenção ela terá

13. Se esta criança estiver desenvolvendo outra atividade como lendo, colorindo, etc. ela terá

14. Se esta criança estiver em grupo com outras criança, ela terá

$\left(\begin{array}{lll}() & (\text { ) }\end{array}\right.$

$($ ) ( ) ( )

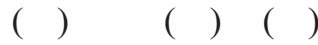

$($ ) ( ) ( )

Figura 1a. Questionário CHAPPS - Children's Auditory Processing Performance Scale (8). 
Condição de escuta - IDEAL:

Se esta criança estiver escutando em uma sala silenciosa, sem distrações, face a face e com bom contato de olho, esta criança tem dificuldade de ouvir e entender (comparada às outras crianças).

Grau de Dificuldade

15. Se for feita uma pergunta para esta criança ela terá.

Menos Mesma Mais

16. Se for dada uma instrução simples para esta criança ela terá.

17. Se for dada uma instrução complexa, múltipla esta criança terá.

$\begin{array}{lll}(\text { ) } & (\text { ) } & (\text { ) } \\ (\text { ) } & (\text { ) } & (\text { ) } \\ (\text { ) } & (\text { ) } & (\text { ) }\end{array}$

\section{Condição de escuta - MÚLTIPLAS INFORMAÇÕES:}

Quando muitos estímulos (por exemplo: visual, tátil, etc.) estão presentes ao mesmo tempo, esta criança tem dificuldade de ouvir e entender (comparada com outra criança).

18. Se esta criança estiver vendo o rosto do falante ela terá

19. Se esta criançã estiver ouvindo algum material que esteja sendo lindo Alto por outra criança, ela terá.

20. Se esta criança estiver ouvindo ou vendo alguém mostrar uma ilustração como um desenho, informação na lousa, ela terá

Grau de Dificuldade
Menos
$\begin{array}{ccc}\text { Mesma Mais } \\ (\text { ) } & (\text { ) } & (\text { ) } \\ (\text { ) } & (\text { ) } \\ (\text { ) } & (\text { ) } & (\text { ) }\end{array}$

Condição de escuta - MEMÓRIA/SEQUÊNCIA AUDITIVA:

Se for solicitado a esta criança para se lembrar de informações faladas, comparada a outras crianças, esta criança:

21. Imediatamente recorda informações assim como uma palavra, soletrar palavras números.

22. Imediatamente lembra-se de instruções simples.

23. Imediatamente lembra-se de instruções complexas.

24. Não somente se lembra da informação, mas também a ordena e a coloca na sequência correta.

25. Consegue se lembrar de palavras ditas há uma hora ou mais.

26. Consegue se lembrar de instruções simples ditas há uma hora ou mais.

27. Consegue se lembrar de instruções complexas ditas há uma hora ou mais.

28. Consegue se lembrar de informações ditas há 24 horas ou mais.

Grau de Dificuldade

Menos Mesma Mais

Condição de escuta - ATENÇÃ̃O AUDITIVA:

Se esta criança precisar prestar atenção, por um longo período, no que está sendo falado, comparado com outras crianças, ela terá:

29. Se estiver escutando por um período menor que 5 minutos ela terá.

30. Se estiver escutando por um período maior que 10 minutos ela terá.

31. Se estiver escutando em uma sala silenciosa ela terá.

32. Se estiver escutando em uma sala barulhenta ela terá.

33. Se estiver escutando as informações pela manhã ela terá.

34. Se estiver escutando no final da tarde ela terá.

$\begin{array}{lll}(\text { ) } & (\text { ) } & (\text { ) } \\ (\text { ) } & (\text { ) } & (\text { ) } \\ (\text { ) } & (\text { ) } & (\text { ) }\end{array}$

35. Se estiver escutando em uma sala onde exita também distrações visuais ela terá.

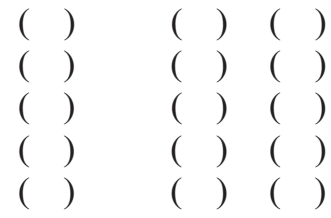

Figura 1b. Questionário CHAPPS - Children's Auditory Processing Performance Scale (8). Grau de Dificuldade Menos Mesma Mais

$\begin{array}{lll}(\quad) & (\quad) & (\quad) \\ () & (\quad) & (\quad) \\ () & (\quad) & (\quad) \\ () & (\quad) & (\quad) \\ () & (\quad) & (\quad) \\ () & (\quad) & (\quad)\end{array}$

$(\quad) \quad(\quad)$ 
Cada uma destas seis condições/funções de escuta possui números de itens diferentes, perfazendo 36 itens em seu total.

Em cada item foi solicitado ao professor julgar graus de dificuldade experenciado pelo escolar com fissura labiopalatina, comparando esta criança com o conhecimento que o professor tem de outras crianças da escola, da mesma idade e similar condição, porém sem esta malformação craniofacial. Para cada grau de dificuldade foi atribuída uma pontuação correspondente: $(+1)$ menos dificuldade, (0) mesma dificuldade e, (-1) mais dificuldade, no qual o professor deveria assinalar a resposta escolhida.

Para analisar as características de escuta no CHAPPS, considerou-se o valor obtido em cada uma das seis condições de escuta (média dos valores ali pontuados) e, o valor obtido no seu total CHAPPS total (soma de todos os itens assinalados divididos por 36). Os resultados obtidos foram analisados verificando-se as respostas separadamente para as variáveis gênero e série escolar, nas seis condições de escuta: no ruído, no silêncio, em situação ideal, na presença de múltiplas informações, quando requerido lembrar a informação ouvida (memória/sequência auditiva) e em longos períodos de escuta (atenção auditiva).

Este estudo foi submetido à Comissão de Ética em Pesquisa e obteve parecer favorável sob o Protocolo $\mathrm{n}^{\circ}$ 041/2003UEPCEP.
Os dados coletados foram digitados em uma planilha do programa Excel (Microsoft Corporation), e posteriormente importados para o Programa Estatístico/Statistica for Window-versão 5.1 Stat Soft. Inc. Além da estatística descritiva (média e desvio padrão), utilizou-se para analisar as diferenças entre gênero, série e condição de escuta a análise de variância a 3 critérios e o Teste de Tukey, sendo adotado nível de significância de 5\% ( $\mathrm{p}<0,05)$.

\section{RESULTADOS}

Com base nos resultados obtidos no questionário CHAPPS, elaborou-se a Tabela 2 e 3 demonstrando a distribuição dos valores médios e desvios-padrão para as crianças amostradas, de acordo com cada série escolar e o gênero, segundo a condição de escuta, respectivamente.

A análise de variância a três critérios não demonstrou diferença estatisticamente significativa entre gêneros ( $p=0,130)$ nem entre séries ( $p=0,555)$, havendo, somente entre as condições de escuta $(\mathrm{p}<0,001)$ (Tabela 4).

\section{DISCUSSÃO}

A análise dos dados deste estudo sugeriu que a média do julgamento das características de escuta dos alunos realizado pelos professores (valor médio de $-0,08$, desvio padrão de 0,27 ), foi muito próxima ao valor zero,

Tabela 2. Valores médios (desvios-padrão) em cada condição de escuta, segundo a série escolar.

\begin{tabular}{|c|c|c|c|c|c|c|c|}
\hline \multirow[t]{2}{*}{ Série } & \multicolumn{5}{|c|}{ Condição de Escuta } & \multirow[b]{2}{*}{ AtençãoAuditiva } & \multirow[b]{2}{*}{ Chapps total } \\
\hline & Ruído & Silêncio & Ideal & Múltiplos Estímulos & Memória Auditiva & & \\
\hline la &,$- 16(0,32)$ &,$- 03(0,33)$ & $0, \mid I(0,4 I)$ & $0,09(0,4 I)$ &,$- \mid 4(0,42)$ &,$- 07(0,26)$ &,$- 06(0,26)$ \\
\hline 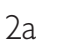 &,$- 17(0,30)$ &,$- 03(0,29)$ & $0,00(0,30)$ & $0,06(0,34)$ &,$- \mid 4(0,37)$ &,$- 09(0,25)$ &,$- 09(0,24)$ \\
\hline За &,$- 17(0,37)$ &,$- 06(0,34)$ & $0,04(0,39)$ & $-0,03(0,42)$ &,$- 21(0,39)$ &,$- 13(0,34)$ &,$- 12(0,30)$ \\
\hline $\mathrm{Ha}$ &,$- 19(0,37)$ &,$- 00(0,29)$ & $0,07(0,34)$ & $-0,00(0,35)$ &,$- 06(0,38)$ &,$- 04(0,32)$ &,$- 06(0,27)$ \\
\hline Id &,$- 17(0,34)$ &,$- 03(0,31)$ & $0,05(0,36)$ & $0,03(0,38)$ &,$- 14(0,39)$ &,$- 09(0,29)$ &,$- 08(0,27)$ \\
\hline
\end{tabular}

$\mathrm{p}=0,555$

Tabela 3. Valores médios e desvios-padrão em cada condição de escuta segundo o gênero (G).

\begin{tabular}{lccccccc}
\hline G & & \multicolumn{6}{c}{ Condição de Escuta } \\
& Ruído & Silêncio & Ideal & Múltiplos Estímulos & MemóriaAuditiva & AtençãoAuditiva & Chapps total \\
\hline Fem & $-0,13(0,36)$ & $-0,00(0,34)$ & $0,08(0,39)$ & $0,06(0,39)$ & $-0,13(0,38)$ & $-0,06(0,30)$ & $-0,05(0,27)$ \\
Mas & $-0,20(0,32)$ & $-0,06(0,30)$ & $0,03(0,34)$ & $0,08(0,38)$ & $-0,15(0,40)$ & $-0,10(0,29)$ & $-0,10(0,27)$ \\
\hline Total & $-0,17(0,34)$ & $-0,03(0,31)$ & $0,05(0,36)$ & $0,03(0,38)$ & $-0,14(0,39)$ & $-0,09(0,29)$ & $-0,08(0,27)$ \\
\hline
\end{tabular}

$p=0,130$ 
Tabela 4. Comparação entre as condições de escuta (teste deTukey).

\begin{tabular}{lcc}
\hline Condição de Escuta & Média & Desvio padrão \\
\hline Ruído & $-0,17$ (a) & 0,34 \\
Silêncio & $-0,03$ (c) & 0,31 \\
Ideal & 0,05 (d) & 0,36 \\
Múltiplos Estímulos & 0,03 (d) & 0,38 \\
MemóriaAuditiva & $-0,14$ (a,b) & 0,39 \\
Atenção Auditiva & $-0,09$ (b,c) & 0,29 \\
\hline
\end{tabular}

(Condição com a mesma letra não possui diferença estatisticamente significativa entre si) $p<0,00$ ।

sinalizando "mesma dificuldade". Assim, por meio deste instrumento utilizado, o professor não identificou diferença entre as características do aluno com fissura labiopalatina com outro sem esta malformação craniofacial, observandoos nas condições de escuta apontadas no questionário. Este achado está em concordância com estudo anteriormente realizado (11), no julgamento realizado pelos pais de crianças com fissura labiopalatina, utilizando o questionário CHAPPS.

No que se refere ao resultado deste estudo, na comparação entre as condições de escuta observadas, as características de escuta das crianças amostradas na condição de escuta na presença de ruído, foi o de maior dificuldade.

Uma das queixas mais comuns de sujeitos com transtorno de processamento auditivo é o problema de processamento sob condições de difícil escuta. Quando avaliados, muitos desses farão muito bem em uma situação favorável. Entretanto, quando os sinais estão distorcidos ou degradados, frequentemente demonstram dificuldades significantes, devido à retirada de alguma das redundâncias intrínsecas do sinal de fala (12).

Um dos métodos de reduzir a redundância de um sinal de fala é introduzindo um ruído de fundo (ruído) juntamente com este sinal. Desta forma, um ouvinte com transtorno de processamento auditivo apresenta dificuldade de reconhecer a fala na presença de ruído (10).

Neste sentido a escola, encontra-se sob forte impacto de ruídos diversos, que se tornam opositores invisíveis à aprendizagem, em um local onde a situação de escuta deveria ser muito privilegiada. Assim, em uma situação desfavorável em que há competição entre a fala do professor e os demais ruídos, o desempenho escolar pode sofrer interferência (13). Pesquisadores (14) consideram que o nível de ruído encontrado nas escolas está acima de valores recomendados, sendo esta uma situação de escuta desfavorável, levando a criança a necessitar de maior atenção para reter a mensagem falada.

Memória auditiva é a habilidade para armazenar e reter o estímulo auditivo. Processo que permite arquivar as informações para poder recuperá-las quando necessário (15). Algumas tarefas de processamento auditivo requerem que a criança retenha informação para formular uma resposta. Assim, a memória auditiva é fundamental para ativar habilidades de escuta (16). A condição de quando solicitada a recordar a informação ouvida (memória auditiva) foi a segunda de maior dificuldade neste estudo.

O estudante com transtorno de processamento auditivo pode demonstrar problemas com compreensão auditiva, discriminação auditiva, memória auditiva, figura-fundo auditiva e atenção auditiva, entre outros (16).

Atenção auditiva é definida como um processo cognitivo que permite o ouvinte focar seletivamente no estímulo de interesse, enquanto ignora um estímulo competitivo não relevante, limitando a quantidade de informação processada ao propósito (17). O aprendizado depende da atenção que está associada com aquilo que é importante, o significado influenciará no grau de atenção (18). A condição de longos períodos de escuta (atenção auditiva), nesta investigação esteve entre as de mais dificuldade apresentada pelo aluno.

Ao se analisar, neste trabalho, a diferença significativa entre os resultados obtidos dos comportamentos reativos frente às condições de escuta no ruído, de quando é requerido lembrar a informação ouvida (memória auditiva) e, em longos períodos de escuta (atenção auditiva), em relação às demais condições, estudos (19) têm demonstrado que a exposição a níveis de ruído elevados pode causar prejuízos, tais como o decréscimo da atenção, porém as crianças podem adaptar-se a interferência do ruído, durante as atividades pela filtragem do estímulo ruidoso indesejável. Elas podem utilizar desta estratégia até quando não existe ruído, levando para sua pobre habilidade em sustentar a atenção na sala de aula, que pode com o tempo continuar a afetar a atenção, mesmo na ausência da exposição ruidosa (Medical Research Council 1997) (20) podendo justificar a dificuldade dos escolares quando também na condição de escuta no silêncio.

Vários aspectos importantes foram verificados nos dados obtidos utilizando este instrumento, em relação à condição de escuta do aluno com fissura labiopalatina, tendo em vista que quando comparado a outro trabalho realizado, (1) no qual foi aplicado testes específicos, confirma problemas de atenção auditiva, memória verbal, histórico de otites recorrentes, alta taxa de distúrbio de aprendizagem, repeti- 
ção e baixa taxa de aproveitamento escolar nesta população.

Os dados encontrados neste estudo sustentam os de estudiosos $(21,22)$ que concluíram que as crianças com fissura labiopalatina apresentaram dificuldade nas habilidades auditivas de figura-fundo e atenção seletiva, por meio dos testes aplicados, sugerindo a inclusão da avaliação do processamento auditivo na bateria auditiva clínica de rotina para os sujeitos com esta malformação.

O julgamento dos professores a respeito das condições de escuta apresentadas pelo aluno, como tendo mais dificuldades (no ruído, na memória e na atenção auditiva) sugere que as crianças estudadas devam ser submetidas a uma avaliação especializada do processamento auditivo, tendo em vista que as alterações encontradas são comuns em crianças ou adultos com alteração de processamento auditivo, podendo a criança não conseguir interpretar o som, uma vez que essa interpretação depende das habilidades auditivas organizadas e estruturadas ligadas às funções cerebrais como atenção e memória.

Desta forma, a inclusão de procedimentos, tais como, questionários, checklist na investigação de alterações do processamento das informações auditivas, na bateria audiológica clínica de rotina para os sujeitos com fissura labiopalatina, parece se justificar para uma melhor orientação no processo diagnóstico, terapêutica e familiar, visando não só melhorias no desempenho acadêmico, como também uma melhor qualidade de vida. Tais procedimentos podem dar informações valiosas, em relação ao real impacto das alterações auditivas, ajudando no processo de diagnóstico diferencial, entretanto não devem ser superestimados, nem tão pouco utilizados com propósitos de diagnósticos.

\section{CONCLUSÃO}

Neste estudo a escuta de crianças com fissura labiopalatina, segundo o julgamento do professor ao de outra criança de mesma idade e condição de escuta semelhante, não portadora desta malformação craniofacial conforme avalia o CHAPPS, mostrou-se, praticamente, similar. Entretanto apresentaram condição de mais dificuldade no ruído, quando solicitadas a recordarem a informação ouvida (memória auditiva) e durante longo período de escuta (atenção auditiva), para qualquer um dos gêneros, como para todas as séries escolares frequentadas.

\section{REFERÊNCIAS BIBLIOGRÁFICAS}

1. Minardi CG, Souza AC, Netto MP, Ulhôa FM, Feniman MR, Campos CF et al. Auditory abilities in children with cleft lip and/or palate according to Fishers. Acta Otorrinolaringol Esp. 2004, 55(4):160-4.

2. Boscariol M, André KD, Feniman MR. Crianças com fissura isolada de palato: desempenho nos testes de processamento auditivo. Rev Bras Otorrinolaringol. 2009, 75(2):213-20.

3. Jerger J, Musiek F. Report of the Consensus Conference on the Diagnosis of Auditory Processing Disorders in SchoolAged Children. J Am Acad Audiol. 2000, 11:467-74.

4. American Speech-Language-Hearing Association. (2005). (Central) Auditory Processing Disorders. Disponível em: URL: http://www.asha.org/members/deskref-journals/deskref/ default. Acesso em 22 de fevereiro de 2008.

5. Bueno C. Crianças com dificuldades na escola: onde mora o problema? Cienc Cult. 2008, 60(2):11-3.

6. King WM. Comorbid auditory processing disorder in developmental dyslexia. Ear Hear. 2003, 24(5):448-56.

7. Sauer L, Pereira LD, Ciasca SM, Pestun M, Guerreiro MM. Processamento auditivo e SPECT em crianças com dislexia. Arq Neuro-Psiquiatr. 2006, 64(1):108-11.

8. Smoski WJ, Brunt MA, Tannahill JC. Listening characteristics of children with central auditory processing disorders. Lang Speech Hear Serv Sch. 1992, 23:145-52.

9. Musiek FE, Guerkink NA. Auditory perceptual problems in children: considerations for the otolaryngologist and audiologist. Laryngoscope. 1980, 90:962-70.

10. Momensohn-Santos MT, Branco-Barreiro FCA. Avaliação e intervenção fonoaudiológica no transtorno de processamento auditivo. In: Ferreira LP, Befi-Lopes DM, Limongi SCO, organizador. Tratado de Fonoaudiologia. São Paulo: Roca; 2004. p.553-68.

11. Barufi L, Netto MP, Ulhoa FM, Rego CF, Feniman MR, Cruz MS et al. Comportamento de escuta em sujeitos com fissura labiopalatina: achados preliminares. J Bras Fonoaudiol. 2004, 5:91-5.

12. Bellis T. Interpretation of APD test results In: Parthasarathy TK, editor. An introduction to auditory processing disorders in children. Mahwah: LEA; 2006. p.145-60.

13. Dreossi RCF, Momensohn-Santos T. O Ruído e sua interferência sobre estudantes em uma sala de aula: revisão de literatura. Pró-Fono. 2005, 17(2):251-8.

14. Jaroszewski GC, Zeigelboim BS, Lacerda A. Ruído escolar 
e sua implicação na atividade de ditado. Rev Cefac. 2007, $9(1): 122-32$.

15. Izquierdo I, Vianna MRM, Cammarota M, Izquierdo LA. Mecanismos da memória. Scie Am Bras. 2003, 2(17):99104.

16. Kelly DA. Suggestions for parents, teachers, speechlanguage pathologists, and students: enhancing functional outcomes in children with APD. In: Parthasarathy TK, editor. An introduction to auditory processing disorders in children. Mahwah: LEA; 2006. p.229-45.

17. Medwetsky L. Central auditory processing. In: Katz J, editor. Handbook of Clinical Audiology. 5th ed. New York: Lippincott Willian \& Wilkins; 2002. p.495-509.

18. Gray HM, Ambady N, Lowenthal WT, Deldin P. P300 as an index of attention to self-relevant stimuli. J Exp Soc Psychol. 2004, 40(2):216-24.
19. Ristovska G, Gjorgjev D, Pop Jordanova N. Psychosocial effects of community noise: cross sectional study of school children in urban center of Skopje, Macedonia. Croat Med J. 2004, 45:473-6.

20. Medical Research Council. IEH Report R10. The nonauditory effects of noise. Leicester, UK: Institute for Environment and Health; 1997.

21. Campos CF, Cruz MS, Feniman MR. Habilidades auditivas de figura-fundo e atenção seletiva em crianças portadoras de fissura labiopalatina. In: Anais do $1^{\circ}$ Congresso Brasileiro de Fonoaudiologia e Genética dos Distúrbios da Comunicação; 2002 25-27 de mar; Fortaleza, Brasil. Fortaleza: Fundação Edson Queiroz, Universidade de Fortaleza; 2002. p.44.

22. Cruz MS, Campos CF, Feniman MR. In: Anais da $9^{\underline{a}}$ Jornada Fonoaudiológica Dra. Mariza Ribeiro Feniman; 2002 28-31 ago; Bauru, Brasil: Faculdade de Odontologia de Bauru, Universidade de São Paulo; 2002.p.26. 